\title{
Successful treatment of a bowel fistula in the open abdomen by perforator flaps and an aponeurosis plug
}

\author{
Yasunori Sashida ${ }^{1}$, Munefumi Kayo ${ }^{2}$, Hironobu Hachiman ${ }^{2}$, Kazuki Hori², Yukihiro Kanda ${ }^{2}$, \\ Akihiro Nagoya ${ }^{3}$ \\ Departments of ${ }^{1}$ Plastic Surgery and ${ }^{2}$ Surgery, Okinawa Prefectural Hokubu Hospital, Nago; ${ }^{3}$ National Hospital Organization, Kinki-chuo \\ Chest Medical Center, Sakai, Japan
}

\begin{abstract}
In this report, we present a case of successful treatment of a bowel fistula in the open abdomen by perforator flaps and an aponeurosis plug. A 70-year-old man underwent total gastrectomy and developed anastomotic leakage and dehiscence of the abdominal wound a week later. He was dependent upon extracorporeal membrane oxygenation, continuous hemodiafiltration, and a respirator. Bowel fluids contaminated the open abdomen. Two months after the gastric operation, a plastic surgery team, in consultation with general surgeons, performed perforator flaps on both sides and constructed, as it were, a bridge of skin sealing the orifice of the fistula. The aponeurosis of the external oblique muscle was elevated with the flap to be used as a plug. The perforators of the flaps were identified on preoperative and intraoperative ultrasonography. This modality allowed us to locate the perforators precisely and to evaluate the perforators by assessing their diameters and performing a waveform analysis. The contamination decreased dramatically afterwards. The bare areas were gradually covered by skin grafts. The fistula was closed completely 18 days after the perforator flap. An ultrasoundguided perforator flap with an aponeurosis plug can be an option for patients suffering from an open abdomen with a bowel fistula.
\end{abstract}

Keywords Intestinal fistula / Perforator flap / Ultrasonography, Doppler / Abdominal wound closure techniques
Correspondence: Yasunori Sashida Department of Plastic Surgery, Okinawa Prefectural Hokubu Hospital, Ikyoku 2-12-3 Onaka, Nago 905-8512, Japan

Tel: $+81-980522719$

Fax: $+81-980542298$

E-mail: sashida@cosmos.ne.jp
This article contains Supplemental Videos S1 and S2.

\section{INTRODUCTION}

A bowel fistula in the open abdomen, which usually takes place in very serious situations such as septic shock, severe trauma, or multiple organ failure, is a challenge even for experienced surgeons. In most instances, surgeons cannot bring the patient into the operating room because the patient is critically ill; in fact, the mortality rate has been reported to be as high as $67 \%$ [1]. Herein, a case of successful treatment of this condition using perforator flaps is reported. Ultrasonography played a very important role in identifying perforators in ideal locations to elevate the appropriate flaps. 


\section{CASE}

A 70-year-old man was referred to the plastic surgery team for consultation because of a bowel fistula in the open abdomen 2 months after total gastrectomy for stomach cancer. He had undergone several abdominal operations, including abscess drainage and closure of the abdominal wall with absorbable mesh, and had just become free from the aid of extracorporeal membrane oxygenation, which was started when he was in severe respiratory failure and septic shock. As abdominal computed tomography showed that contrast medium in the small bowel was scattered throughout his open abdomen, it was concluded that bowel fluids were continuously contaminating his open abdomen, despite frequent dressing changes (Fig. 1A, Supplemental Video S1). At the time of consultation, he was still on a ventilator, was undergoing continuous hemodiafiltration, and was malnourished, with a low serum albumin level of $1.1 \mathrm{~g} / \mathrm{dL}$. The general surgeons decided that a radical operation such as a reanastomosis of the small bowels would be too risky because the patient was too critically ill to undergo successful anastomosis or exteriorization of the bowels, which are usually very fragile in the open abdomen and difficult to expose without injury. Instead, the surgeons proposed the possibility of a perforator flap elevated from the skin near the fistula. The surgeons suggested that if the output from the fistula could be decreased by coverage and any area of the open abdomen could be made free from contamination, that would potentially allow the open area to be decreased gradually by skin grafts. Using ultrasound, a perfora- tor reliable enough for a perforator flap to be elevated was identified in the right lower skin near the fistula in the open abdomen. Its diameter was $1.06 \mathrm{~mm}$ (Fig. 1B, Supplemental Video S1) as assessed using the e-flow mode of the ProSound F75 (Hitachi Ltd., Tokyo, Japan) and a steep systolic crest was noted in its waveform, forming a notch between the systolic and diastolic waves with a peak systolic velocity of $52.9 \mathrm{~cm} / \mathrm{sec}$ (Fig. 1C). The patient and his family consented to the operation. The study was approved by the Institutional Review Board of Okinawa Prefectural Hokubu Hospital (IRB No. 2016010) and performed in accordance with the principles of the Declaration of Helsinki. Written informed consents were obtained.

In the operating room, in the right lower abdomen, a $9 \times 26$ $\mathrm{cm}$ perforator flap was elevated with the anterior sheath of the rectus abdominis muscle (Fig. 2A). This flap was based on the perforator that was previously identified using ultrasonography (Supplemental Video S1). Additionally, a part of the aponeurosis of the external oblique muscle was elevated with the anterior sheath, packed into the orifice of the fistula as a plug, and sutured to the wall of the small bowel very gently (Fig. 2B, Supplemental Video S2). To keep the orifice of the fistula plugged continuously, it was necessary to elevate another flap on the contralateral side of the skin and suture it to the first flap to make, as it were, a stout bridge (Figs. 2B, 3A). Intraoperative ultrasonography was used to identify another perforator in the left upper skin by the side of the open abdomen (Fig. 2B, C). Its diameter was $0.85 \mathrm{~mm}$, as assessed in the e-flow mode of the ProSound $\alpha 7$ (Hitachi Ltd.), and a systolic steep crest was noted in its wave-

Fig. 1. Preoperative clinical photos and ultrasound images

(A) Fistula orifice (green triangle) in the open abdomen. Ultrasound could detect a perforator (red dot) in the right lower abdomen. (B) A perforator in the ultrasound image (red arrow). The diameter of the perforator was measured as $1.06 \mathrm{~mm}$ (red circle). (C) Pulsed Doppler mode image of the perforator obtained from a perforator as suggested by yellow arrow in (A). A systolic steep crest was noted in its waveform (white arrow), forming a notch between the systolic and diastolic waves with a peak systolic velocity of $52.9 \mathrm{~cm} / \mathrm{sec}$ (red circle).

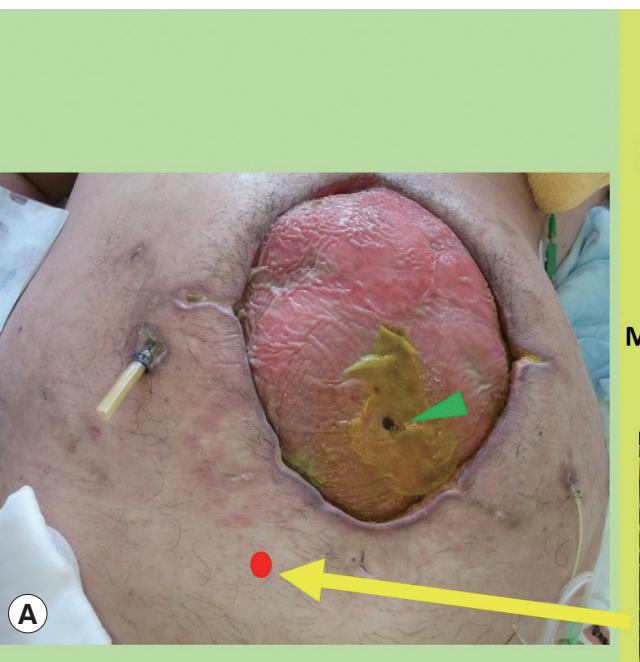

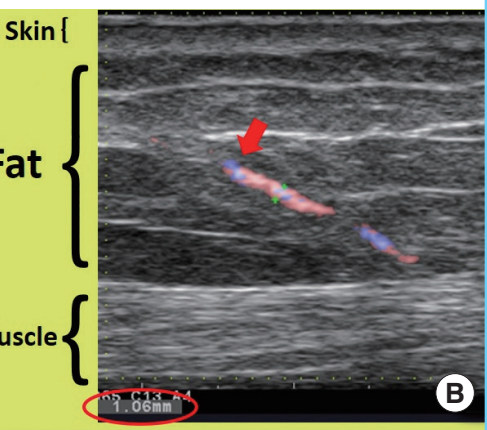

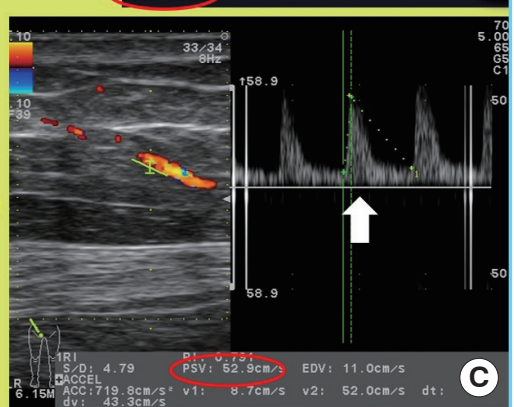




\section{Fig. 2. Clinical photographs and schema of the operation}

(A) In a clinical photograph, a $9 \times 26 \mathrm{~cm}$ perforator flap (green triangles) was designed in the right lower skin based on a perforator (red dot) that was identified by preoperative ultrasonography. (B) The operative procedures are schematically presented. The aponeurosis of the external oblique muscle (white triangles), harvested with the perforator flap, was packed into the bowel fistula (green triangle) and was sutured very gently. In the blue rectangle with an arrow at the middle-bottom of the figure, the method of sealing and suturing is presented in detail. Another $5 \times 12 \mathrm{~cm}$ flap (**) was elevated based upon a perforator that was identified by intraoperative ultrasonography in the upper-left skin area. Its tip was approximated to the tip of the first flap $\left(^{*}\right)$ to make, as it were, a stout bridge to keep the orifice of the fistula sealed continuously. (C) As shown in a clinical photograph, the second flap was designed (green triangles) in the left upper skin area, including a perforator (red dot), identified by intraoperative ultrasonography. The aponeurosis of the external oblique muscle (white triangle) was used to plug the fistula orifice.
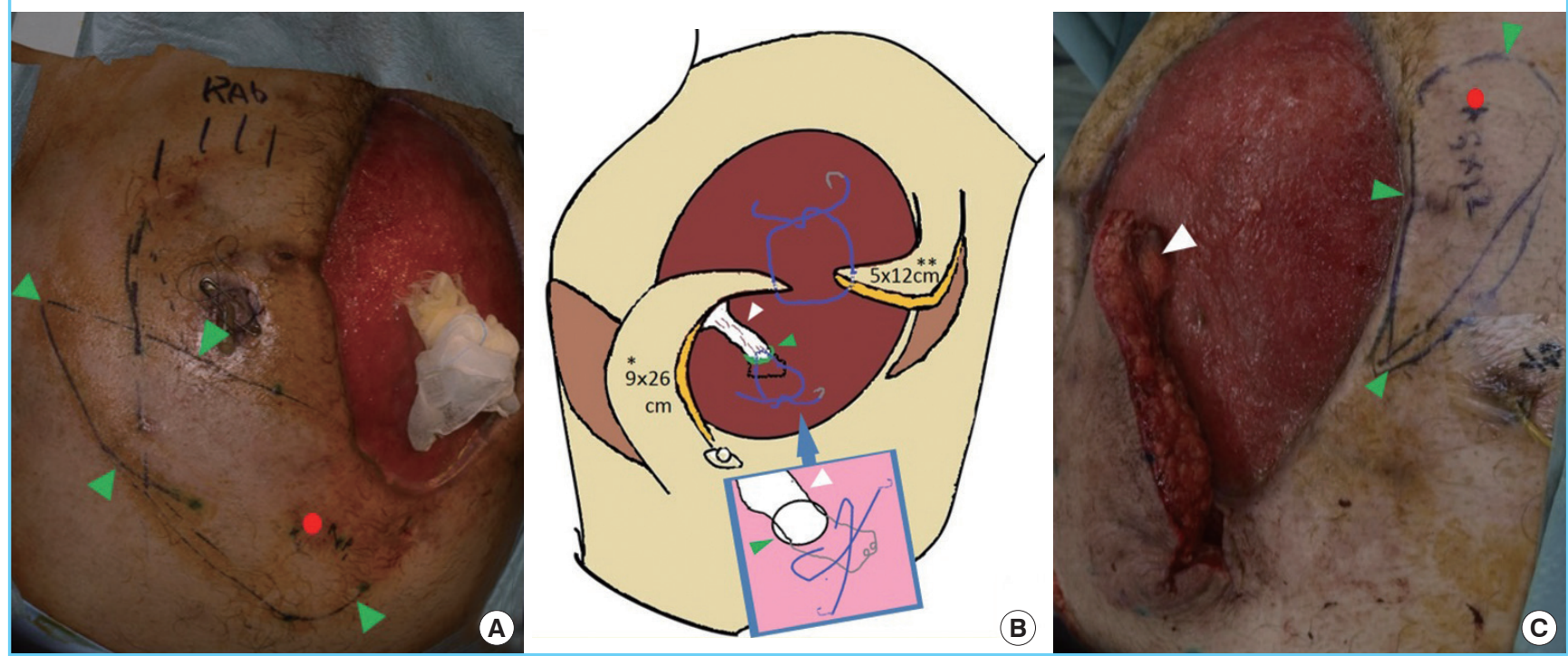

\section{Fig. 3. Clinical photographs of the abdomen}

(A) Clinical photograph of the operation, in which the tips of the 2 perforator flaps were sutured (green triangle) so that the orifice of the fistula (yellow dot) could be kept sealed by the flap. In the upper area of the open abdomen, skin grafts can be seen. (B) Clinical photograph taken 6 months after the operation. Note that no open wound was left because the donor sites of the perforator flaps were covered by skin grafts. A colostomy was performed for reconstruction due to colonic obstruction (green triangle).
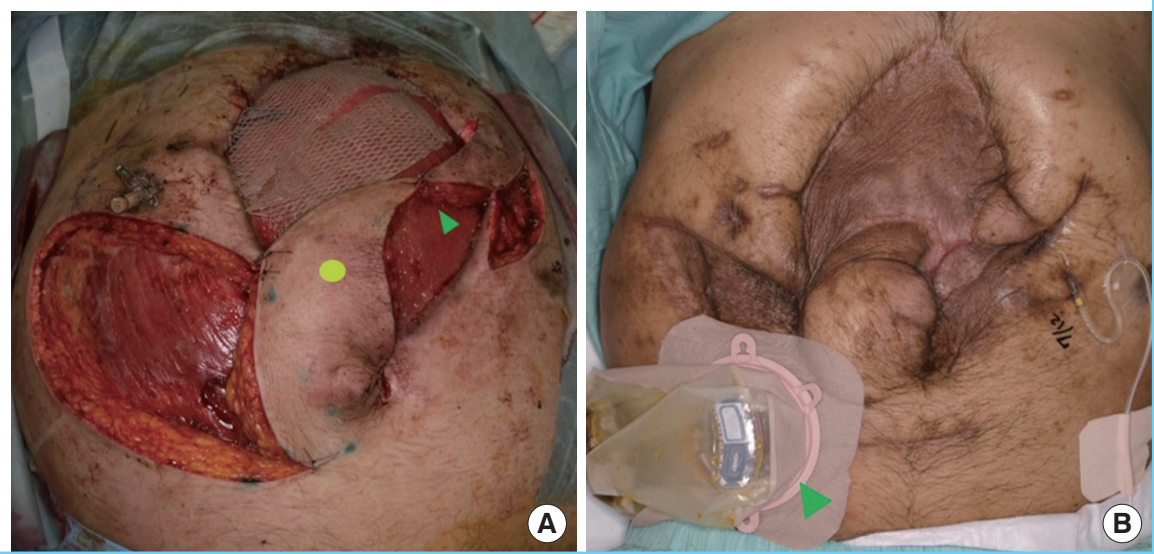

form, forming a notch between the systolic and diastolic waves with a peak systolic velocity of $20.0 \mathrm{~cm} / \mathrm{sec}$. A $5 \times 12 \mathrm{~cm}$ perforator flap was elevated, transposed, and sutured to the tip of the first flap (Figs. 2B, 3A). The flaps continuously covered the orifice of the fistula. The upper part of the open abdomen was covered by skin grafts harvested from the right thigh (Fig. 3A).

Postoperatively, the amount of bowel fluid leakage from the fistula decreased dramatically, reaching zero at 18 days after the operation. A month after the operation, the other areas of the open abdomen, including the donor sites of the perforator flaps, were covered by skin grafts (Fig. 3B). The patient was discharged from the hospital with ambulant status 7 months after the operation, after recovering from respiratory failure and completing rehabilitation.

\section{DISCUSSION}

A bowel fistula in the open abdomen is usually both the cause and result of critical illness, and frequently prevents radical operations. In such situations, a perforator flap might be possible be- 
cause it is less invasive and stressful for the patient than other surgical procedures, such as bowel anastomosis. Ultrasonography is a very useful modality for elevating a perforator flap in an area near the fistula orifice. Compared with computed tomography, ultrasound is more convenient, with no need to bring the patient to a specific location, less expensive, and does not require contrast medium. The blood flow in a perforator can be increased by nearby wounds or ulcerations [2], which sometimes allows us to identify the perforator and elevate a freestyle perforator flap. Ultrasonography is not only able to identify perforators, but can also be used to evaluate them [3] by their diameter, peak systolic velocity, and waveform pattern [4]. It has been reported that when evaluating the waveform of a small artery, the presence of a systolic steep crest with a notch between the systolic and diastolic waves is important [5]. Assuming that there is no stenotic disease in the small artery, for a given diameter, higher peak systolic velocity is associated with higher average velocity, indicating more abundant blood flow. Fortunately, in this case, two perforators for the flaps were found in ideal locations in the first ultrasonography exams, with no need to evaluate multiple perforators, and both perforators showed good waveforms and could provide the flaps with a sufficient blood supply.

A previous report has described the treatment of a bowel fistula in the open abdomen using large bi-pedicled flaps, which are more invasive than perforator flaps [1]. Although the patient in our case was critically ill, the bowel fistula in the open abdomen was successfully treated by ultrasound-guided perforator flaps and an aponeurosis plug. This method should be considered as an option for this very challenging situation.

\section{NOTES}

\section{Conflict of interest}

No potential conflict of interest relevant to this article was reported.

\section{Ethical approval}

The study was approved by the Institutional Review Board of Okinawa Prefectural Hokubu Hospital (IRB No. 2016010) and performed in accordance with the principles of the Declaration of Helsinki. Written informed consents were obtained.

\section{Patient consent}

The patient provided written informed consent for the publication and the use of his images.

\section{REFERENCES}

1. Sriussadaporn S, Sriussadaporn S, Kritayakirana K, et al. Operative management of small bowel fistulae associated with open abdomen. Asian J Surg 2006;29:1-7.

2. Rubino C, Coscia V, Cavazzuti AM, et al. Haemodynamic enhancement in perforator flaps: the inversion phenomenon and its clinical significance. A study of the relation of blood velocity and flow between pedicle and perforator vessels in perforator flaps. J Plast Reconstr Aesthet Surg 2006; 59:636-43.

3. Sashida Y, Ishida K, Imaizumi A, et al. Usefulness of color Doppler ultrasound in the planning of perforator flaps. J Jpn Soc Reconstr Microsurg 2013;26:1-9.

4. Hirai T, Ohishi H, Kichikawa K, et al. Ultrasonographic screening for arterial occlusive disease in the pelvis and lower extremities. Radiat Med 1998;16:411-6.

5. Ogino A, Onishi K. Vascular waveform analysis of flap-feeding vessels using color Doppler ultrasonography. Plast Surg Int 2014;2014:249670.

Supplemental Video S1. Preoperative ultrasound.

\section{Supplemental data can be found at: https://doi.org/10.5999/aps.2017.00822.v001}

Supplemental Video S2. Operation video.

Supplemental data can be found at: https://doi.org/10.5999/aps.2017.00822.v002 